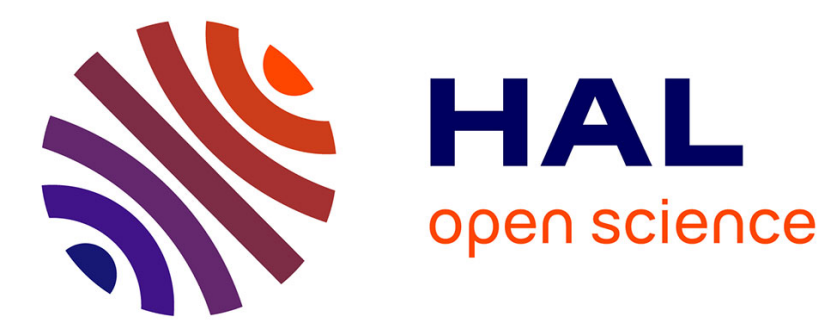

\title{
Des limites de la mémoire historique française de l'esclavage
}

Olivier Pulvar

\section{To cite this version:}

Olivier Pulvar. Des limites de la mémoire historique française de l'esclavage. Cités: Philosophie, politique, Histoire, 2006, pp.186-189. 10.3917/cite.025.0186 . hal-01152967

\section{HAL Id: hal-01152967 \\ https://hal.science/hal-01152967}

Submitted on 18 May 2015

HAL is a multi-disciplinary open access archive for the deposit and dissemination of scientific research documents, whether they are published or not. The documents may come from teaching and research institutions in France or abroad, or from public or private research centers.
L'archive ouverte pluridisciplinaire HAL, est destinée au dépôt et à la diffusion de documents scientifiques de niveau recherche, publiés ou non, émanant des établissements d'enseignement et de recherche français ou étrangers, des laboratoires publics ou privés. 


\section{Des limites de la mémoire historique française de l'esclavage*}

\section{Cités $n^{\circ} 25$, PUF, 2006, p. 186-189.}

Olivier Pulvar ${ }^{* * *}$

Le débat sur la reconnaissance de l'histoire de l'esclavage colonial en France illustre les conflits de mémoire qui émaillent un projet qui se veut unanime. La question de la ritualisation officielle de cette reconnaissance souligne le rôle d'instauration du groupe que joue le travail de mémoire. Dès lors que l'Etat sollicite des figures et mises en scène d'événements présentés comme fondateurs, cherche-t-il moins à légitimer la communauté nationale sur une base idéologique qu'à l'édifier sur le respect de la diversité culturelle ?

La conscience d'appartenance commune que présente toute commémoration se heurte souvent à des lectures différentes de l'histoire officielle. Etudier les tensions qui alimentent une représentation du passé à vocation collective, observer les usages de cette représentation dans la sphère publique, indiquent précisément ce qui pose problème.

\section{Entre amnésie collective et devoir de mémoire}

La commémoration de l'abolition de l'esclavage en France établit d'emblée une hiérarchisation des mémoires par les groupes qui les portent à travers le traitement du thème de la reconnaissance. Les premiers mettent en avant l'acquis républicain de l'abolition pour se faire reconnaître dans le respect des principes fondateurs de la République. Les seconds se réfèrent au crime fondateur de peuples ayant droit à réparation pour se reconnaître dans une identité propre.

Lorsqu'en 1998, la République commémore le cent cinquantenaire de l'abolition définitive de l'esclavage en France, l'événement vante les vertus de la patrie des droits de l'Homme qui a montré l'exemple au reste du monde. A contrario, depuis les années 1960 dans les Outre-mers comme dans l'Hexagone, nombre de mobilisations collectives insistent sur les actes, les lieux et les acteurs des révoltes d'esclaves.

On peut dire que la mémoire historique française de l'esclavage institutionnalise la figure du républicain abolitionniste aux dépends de celle de l'esclave rebelle, portée par des parlementaires d'Outre-mer, des historiens, des artistes ou encore, des responsables associatifs, issus de ces territoires. Les deux personnages n'y remplissent pas la même fonction, comme si prendre en compte le rôle émancipateur du second compromettait la mémoire historique nationale d'une France, patrie des droits de l'homme. ${ }^{1}$

Non seulement le conflit de mémoires provient d'une divergence des approches de situations contemporaines, mais il se structure également autour de mémoires du conflit. Il entre dans des pratiques traditionnelles nationales, l'Etat cherchant toujours à imposer un monopole de la mémoire légitime ${ }^{2}$. Il reste que l'usage de la mémoire amnésique réduit à une domination aveuglante ceux qui l'exercent autant que, l'usage d'une mémoire pour la mémoire enferme dans une domination anesthésiante ceux qui la subissent.

\section{Pourquoi une commémoration nationale ?}

Dans des espaces nationaux de moins en moins homogènes, la dimension mondiale du fait historique de l'esclavage permet certainement de relever le défi actuel du multiculturalisme. Elle offre à la mémoire historique française une visée réconciliatrice qui intègre le passé 
comme «principe d'action pour le présent » et non comme "un fait intransitif, ne conduisant pas au-delà de lui-même »3.

Le caractère officiel et nécessairement national de la commémoration en France pour un événement mondial se justifie alors par :

- la place symbolique du pays des Lumières et des droits de l'Homme dans le fait historique lui-même ;

- la portée exemplaire d'une reconnaissance officielle du crime pour les anciennes métropoles esclavagistes comme pour les anciennes colonies.

C'est à partir d'un retournement de la mémoire en projet que se conçoit h valeur exemplaire extraite de souvenirs traumatisants. Une mémoire qui organise le pardon sans exiger l'oubli peut prendre la forme d'une culture de la considération. Appliquée à la responsabilité politique, elle s'exprime par de la modération, de la mansuétude, de la clémence ; appliquée à la responsabilité morale, elle s'illustre par une volonté de comprendre ces autres dont l'histoire a fait des ennemis. Certains gestes accomplis par des acteurs officiels sur la scène publique saluent cette capacité à partir de laquelle l'action peut se tourner vers l'avenir.

Substituer à l'évocation publique d'approches différentes du passé la mise en public de figures mémorielles partagées, affranchit d'une production identitaire exclusive pour l'engager dans un projet collectif. Le système de représentation dominant qui assume pleinement son passé limite les quêtes identitaires antagonistes.

\section{Sortir d'une domination mortifêre}

Plusieurs auteurs ${ }^{5}$ soulignent le rôle de la République et plus largement celui des Etats démocratiques modernes dans la trajectoire différente de peuples structurée par un rapport inégalitaire. Comment en effet, parler de cohésion sociale sans interroger la cohésion des groupes de cohabitation, elle-même déterminée par l'existence d'un projet social collectif? De quelle nature sont les rapports construits dans un contexte défini entre les membres des différents groupes concernés? L'ordre colonial, fondé sur l'universalisme symbolique ici, justifié économiquement là, est-il seulement remis en cause par quelque évolution formelle dont les effets annoncés ne s'imposent pas mécaniquement ?

Depuis la $3^{\text {ème }}$ République en France, un dispositif social et institutionnel aide à construire le rapport au colonisé par le biais d'un imaginaire colonial ${ }^{6}$. Certes, la liberté dont disposent les individus de construire une réalité à partir de codes et répertoires communs ne signifie nullement qu'ils soient idéologiquement acquis au projet colonial valorisé par le corps social. Mais l'ordre colonial est d'autant mieux accepté qu'il est vécu comme bienfaiteur et que, la domination qu'il exerce est naturalisée.

C'est bien pourquoi un usage de la mémoire non prisonnière du traumatisme libère de la domination. L'exercice demande de renoncer aux hégémonies imposées à l'humanité plutôt que de chercher à renverser le rapport de force. Il se fonde davantage sur un imaginaire de la diversité, né du croisement de visions idiosyncrasiques du réel. Tout support sollicité entre dans un processus de revalorisation de soi-même et, encourage le dialogue interculturel. ${ }^{7}$ 


\section{Les démocraties au risque de l'image}

De toute évidence, l'esclavage colonial peut être relaté en France. Toutefois, l'énonciateur reste limité dans la part intransmissible de l'expérience extrême ; toute forme de récit présente une dimension sélective. C'est là que l'imagination qui montre constitue un piège de l'imaginaire ${ }^{8}$ pour la mémoire.

Dans les espaces culturels hétérogènes, l'appel au passé demeure l'une des dimensions mise en récit pour construire les identités. Les enjeux liés aux choix de mémoire se densifient et favorisent des appropriations de l'histoire produisant ces énonciations identitaires multiples. Pour autant, le récit historique n'est pas le récit de fiction et, le contrat qui lie l'historien à celui qui use de ses recherches n'est pas celui que passe le romancier avec son lecteur.

D'un autre côté, la médiatisation du fait remémoré intervient dans la lecture du passé (comme du présent) en tant qu'elle participe du faire histoire, et qu'elle témoigne de l'événement. Les médias interviennent dans les constructions identitaires en diffusant la mémoire collective. Ainsi, le témoignage médiatique dans son rapport à l'histoire ${ }^{9}$ pose moins la question de son auteur que celle du vecteur d'énonciation. Le média qui montre le témoignage tend à s'instaurer source de la vérité historique.

A la suite de Foucault pour lequel nommer les choses c'est déjà les instrumentaliser et, de Habermas évoquant la légitimation de la discussion publique, l'analyse pose au moins deux problèmes à résoudre pour les démocraties modernes. Qu'advient-il de la lisibilité de la représentation historienne quand l'histoire se fictionalise ? Que devient le débat démocratique quand le récit répond aux exigences de la médiatisation?

\footnotetext{
* Ce texte est tiré d'un Séminaire présenté à l'occasion d'un Colloque international sur la communication pour le développement organisé par l'Université de Douala du 26 au 30 avril 2005 au Cameroun. Il reprend en les développant des éléments de réflexion parus dans l'édition de Martinique du quotidien régional France-Antilles du 28-29 mai 2005, p. 8.

** Olivier Pulvar est Maître de conférences à l'Université Antilles-Guyane, et membre du Groupe d'Etudes et de Recherches en Es pace Créolophone et Francophone. Chercheur associé au Laboratoire Communication et Politique du CNRS (Paris), ses travaux portent sur les transformations sociales des mondes créoles en liaison avec le développement généralisé des phénomènes d'information et de communication.

${ }^{1}$ Sarah Gensburger développe cette approche dans «Les figures du juste et du résistant et l'évolution de la mémoire historique française de l'occupation », Revue française de science politique, vol. 52, $\mathrm{n}^{\circ} 2-3$, avril-juin 2002, p. 291-322.

${ }^{2}$ Joël Candau, Anthropologie de la mémoire, PUF, QSJ, 1996, 127 p. L'expression reprise ici conclut un développement de l'auteur (p. 72-76).

3 Tzvetan Todorov, Les abus de la mémoire, Paris, Arléa, 1998, 61 p. Todorov fait cette distinction entre mémoire exemplaire et mémoire littérale (p. 28-33).

${ }^{4}$ Paul Ricoeur, La mémoire, l'histoire, l'oubli, Paris, Editions du Seuil, 2000, 675 p. Sur ce point, on se reportera p. 637-642.

5 Juliette Sméralda-Amon, La racisation des relations intergroupes ou la problématique de la couleur. Le cas de la Martinique, Paris, L’Harmattan, 2002, 526 p. Christine Chivallon, «Le poids de la différence », Hermès, $\mathrm{n}^{\circ}$ 40, 2004, p. 216-219.

6 Eric Savarèse, L'ordre colonial et sa légitimation en France métropolitaine. Oublier l'autre, Paris, L'Harmattan, 1998, 300 p.

${ }^{7}$ Le courant de la Créolisation, représenté par Edouard Glissant notamment, défend cette thèse.

${ }^{8}$ Paul Ricoeur évoque dans son ouvrage déjà cité, l'imagination à mi-chemin de la fiction et de l'hallucination (p. 64-66). Plus loin, il rappelle que dans le champ politique, le rapport entre justification du pouvoir et prestiges de l'image, persiste même pour les citoyens d'une démocratie qui croient avoir rompu avec l'éloge du roi (p. 344-358).

9 Sandie Scozzi, «La tribune télévisuelle des témoins de la Shoah à l'heure de l'événement judiciaire », communication au $7^{\text {’ème }}$ colloque Brésil-France, Intercom / S.F.S.I.C., Porto Alegre, 30-31 août 2004, 10 p.
} 
